J. Math. Kyoto Univ. JMKYAZ

15-1 (1975)25-52

\title{
Higher differential algebras of discrete valuation rings
}

\author{
By \\ Satoshi SuzUki and Jun-ichi Nishimura
}

(Reccived Dec. 21, 1973)

\section{Introduction.}

The notion of the universal higher differential algebras of commutative rings was defined by Berger in [1] and KawaharaYokoyama in [6] and they developed the general theory on them.

The purpose of the present paper is to discuss properties of complete discrete valuation rings of unequal characteristic, concerned with higher derivations, using above notion. Important results in this paper are Theorem 6.1 and Theorem 7.1. In Theorem 6.1 we prove that five fine properties of complete discrete valuation rings of unequal characteristic related to higher differentials are equivalent to each other and by means of it we can classify those rings into two classes, differentially good and differentially bad. The differentially good class contains all tamely ramified ones and those with perfect residue fields. Although in this theorem the equivalence among (i), (ii) and (iii) and that between (iv) and (v) are proven in routine ways and analogous to facts in differentials of order 1 (Neggers [7], Suzuki [10], [11]), the analogy of the equivalence between these two groups of statements are not true in case of order 1. In Theorem 7.1, it is proven that if a valuation ring $R$ is in the differentially good class, the ideals $\left(f^{\prime}(\pi), \frac{f^{\prime \prime}(\pi)}{2 !}, \ldots, \frac{f^{(n)}}{n !}(\pi)\right.$ ) are independent of the choice of a 
coefficient ring of $R$ and a prime element $\pi$ for $n=1,2, \ldots$, where $f$ is the Eisenstein polynomial over this coefficient ring, satisfying $f(\pi)=0$. In case of differentials of order 1 , we proved an analogous fact in [10], that is, if $R$ satisfies conditions analogous to (iv) and (v) in Theorem 6.1, the ideal $\left(f^{\prime}\right)$ is invariant. We also proved the converse of this fact in [11]. However, the converse problem of Theorem 7.1 itself is still open.

A fairly large portion of the present paper ( $\S 1, \S 2$ and $\S 3$ ) is devoted to studying basic properties of higher differential algebras. Especially, in order to make up the lack of basic discussions on differentials of unramified regular local rings of unequal characteristic in [1] and [6], we start with a general argument on differentials of formally smooth algebras. In these parts we use a definition of higher derivations by Heerema in [3], which are higher derivations with multi-indices, for the sake of generalization.

As an effective tool to discuss differentials of complete discrete valuation rings of unequal characteristic, we extend in $\S 5$ the notion of Neggers' number appeared in [7], [10] and [11].

Throughout this paper, all rings will be assumed to be commutative and have identities. The term "discrete valuation rings" will refer exclusively to discrete valuation rings of rank one.

\section{Generalities.}

Let $\mathbf{Z}$ be the set of integers. Let $\mathbf{N}_{0}$ be the set of nonnegative integers.

Let $\boldsymbol{a}=\left(\alpha_{1}, \ldots, \alpha_{m}\right)$ and $\boldsymbol{b}=\left(\beta_{1}, \ldots, \beta_{m}\right)$ be elements in $\mathbf{Z}^{m}=$ $\mathbf{Z} \times \ldots \times \mathbf{Z}$, and let $h$ be an integer. We define $\boldsymbol{a}+\boldsymbol{b}=\left(\alpha_{1}+\beta_{1}\right.$, $\left.\ldots, \alpha_{m}+\beta_{m}\right)$ and $h \boldsymbol{a}=\left(h \alpha_{1}, \ldots, h \alpha_{m}\right) . \quad \boldsymbol{a} \leq \boldsymbol{b}$ means that $\alpha_{i} \leq \beta_{i}$ for all $i=1,2, \ldots, m$. We denote $\boldsymbol{o}=(0, \ldots, 0)$ and $|\boldsymbol{a}|=\sum_{i=1}^{m} \alpha_{i}$.

Definition 1. 1. $A$ subset $A$ of $\mathbf{N}_{0}{ }^{m} \subset \mathbf{Z}^{m}$ is called an index domain if the following conditions are satisfied.

i) $\Lambda \neq \phi$. 
ii) If $\boldsymbol{a} \in \Lambda, \boldsymbol{b} \in \mathbf{N}_{0}{ }^{m}$ and $\boldsymbol{b} \leq \boldsymbol{a}$, then $\boldsymbol{b} \in \Lambda$.

Let $P$ be a ring. Let $R$ and $S$ be $P$-algebras.

Definition 1.2. (Heerema [3]). $A$ sequence of $P$-linear maps $\partial=\left\{\partial^{a}\right\}_{a \in A}$ of $R$ into $S$ is called a higher $P$-derivation with index domain $A$, if $\partial$ satisfies the following conditions.

i) $\partial^{\circ} 1=1$.

ii) $\partial^{a}(x y)=\sum_{\substack{b+c=a \\ b, c \in A}}^{\sum} \partial^{b} x . \partial^{c} y$, for all $x, y \in R$ and $\boldsymbol{a} \in \mathcal{A}$.

In Definition 1. 2, $\partial^{\circ}$ becomes a $P$-algebra homomorphism and we can define an $R$-algebra structure in $S$ through $\partial^{\circ}$. In case $P=\mathbf{Z}$. we simply call $\partial$ a higher derivation of $R$ into $S$ with index domain $A$. In case $m=1$ and $A=\mathbf{N}_{0}$, we omit "with index domain $A^{\prime \prime}$.

Let $t_{1}, \ldots, t_{m}$ be indeterminates. If $\boldsymbol{a}=\left(\alpha_{1}, \ldots, \alpha_{m}\right) \in \mathbf{N}_{0}^{m}, t^{a}$ denotes the monomial $t_{1}^{\alpha_{1}} \ldots t_{m}^{\alpha_{m}}$. By $\Im_{1}$ we denote an ideal of the power series ring $S\left[\left[t_{1}, \ldots, t_{m}\right]\right]$ generated by all monomials $t^{a}$ with $\boldsymbol{a} \notin A$. Let $\varphi: R \longrightarrow S\left[\left[t_{1}, \ldots, t_{m}\right]\right] / \widetilde{\Im}_{A}$ be a $P$-linear map. We write as

$$
\varphi(x)=\sum_{a \in A}\left(\partial^{a} x\right) \bar{t}^{a} \text { with } \partial^{a} x \in S \text { for } x \in R,
$$

where $\bar{t}_{i}$ denotes the class of $t_{i}$ modulo $\Im_{A}$ for each $i=1,2, \ldots$, $m$. Then $\partial=\left\{\hat{o}^{a}\right\}_{a \in A}$ is a higher $P$-derivation of $R$ into $S$ with index domain $A$ if and only if $\varphi$ is a $P$-algebra homomorphism.

Definition 1. 3. Let $A$ be a $P$-algebra. Let $d=\left\{d^{a}\right\}_{a \in A}$ be a higher $P$-derivation of $R$ into $A$. $A$ is called the higher differential algebra of $R$ over $P$ with index domain $A$ and $d$ is called the canonical higher derivation and we denote $A=A_{P}^{A}(R), d=d_{R / P}$ and $d^{a}=\mathrm{d}_{R / P}^{a}$, if they have the following universal property:

For every higher $P$-derivation $\partial=\left\{\hat{\sigma}^{a}\right\}_{a \in A}$ of $R$ into an arbitrary $P$-algebra $S$ with index domain $A$, there exists a unique $P$-algebra homomorphism $f: A \longrightarrow S$ such that $\partial^{a}=f \circ d^{a}$ for every $\boldsymbol{a} \in \mathcal{A}$.

When we refer to an $R$-algebra structure of $A_{P}^{A}(R)$, we always 
mean that the structure homomorphism is $d_{R / P}^{\circ}$.

In case $P=\mathbf{Z}$, we simply write as $A^{A}(R), d_{R}$ and $d_{R}^{a}$ instead of $A_{P}^{A}(R), d_{R / P}$ and $d_{R / P}^{a}$, respectively. In case $m=1$ and $\Lambda=\mathbf{N}_{0}$, we write as $A_{P}(R)$ instead of $A_{P}^{A}(R)$.

Let $\varphi: R \longrightarrow R^{\prime}$ be a $P$-algebra homomorphism, then $\varphi$ induces canonically a $P$-algebra homomorphism :

$$
A_{P}^{A}(R) \longrightarrow A_{P}^{A}\left(R^{\prime}\right) .
$$

For the proof of each statement in Proposition 1.1, we refer to Berger [1] and Kawahara-Yokoyama [6].

Proposition 1. 1. 1) $A_{P}^{A}(R)$ always exists and is determined uniquely up to $R$-algebra isomorphisms.

2) $A_{P}^{A}(R)$ is a multi-graded $R$-algebra. $A_{P}^{A}(R)=\bigoplus_{a \in \mathrm{N}_{0}^{m}} A_{P}^{a}(R)$, where if $x \in A_{p}^{a}(R)$ and $y \in A_{p}^{b}(R)$, then $x y \in A_{p}^{a+b}(R), R$ is isomorphic to $A_{P}^{\circ}(R)$ and $A_{P}^{a}(R)$ is the $R$-submodule of $A_{P}^{A}(R)$ generated by the elements $\left(d^{a_{1}} x_{1}\right) \ldots\left(d^{a_{r}} x_{r}\right)$ such that $x_{1}, \ldots, x_{r} \in R, \boldsymbol{a}_{1}, \ldots, \boldsymbol{a}_{r} \in \Lambda-\boldsymbol{o}$ and $\boldsymbol{a}_{1}+\ldots+\boldsymbol{a}_{r}=\boldsymbol{a}$ for $\boldsymbol{a} \neq \boldsymbol{o}$.

3) If $e_{i}=(0, \ldots, 0,1,0, \ldots, 0)$ is in $A$, then $A_{P}^{{ }^{i}}(R)$ $\simeq \Omega_{R / P}^{1}(=$ the module of $P$-differential of $R)$.

4) If $\mathfrak{U}$ is an ideal of $R$ and $\bar{R}=R / \mathfrak{A}$, then we have $A_{P}^{A}(\bar{R}) \simeq$ $A_{P}^{A}(R) / \mathfrak{Y}$, where $\mathfrak{B}$ is the ideal of $A_{P}^{A}(R)$ generated by the elements $d_{R / P}^{a} x$ with $x \in \mathfrak{U}$ and $a \in \Lambda$.

5) If $R$ is a $P_{1}$-algebra and $P_{1}$ is a $P$-algebra, then we have $A_{P_{1}}^{A}$ $(R) \simeq A_{P}^{A}(R) / \mathfrak{W}$, where $\mathfrak{W}$ is the ideal of $A_{P}^{A}(R)$ generated by the elements $d_{R / P}^{a} y$ with $y \in P_{1}$ and $\boldsymbol{a} \in \Lambda_{-}$o.

6) If $R_{1}$ and $R_{2}$ are $P$-algebras, then we have

$$
A_{P}^{A}\left(R_{1} \otimes_{P} R_{2}\right) \simeq A_{P}^{A}\left(R_{1}\right) \bigotimes_{P} A_{P}^{A}\left(R_{2}\right) .
$$

Moreover, it holds that

$$
A_{P}^{a}\left(R_{1} \otimes_{P} R_{2}\right) \simeq \bigoplus_{b+c=a} A_{P}^{b}\left(R_{1}\right) \otimes_{P} A_{P}^{c}\left(R_{2}\right) \text { for every } a \in \mathbf{N}_{0}^{m} .
$$

7) If $\left\{R_{\alpha}\right\}_{\alpha \in \Gamma}$ is a direct system of $P$-algebras and $R=\underset{a}{\lim } R_{\alpha}$, 
it holds that $\left\{A_{P}^{A}\left(R_{\alpha}\right)\right\}_{\alpha \in \Gamma}$ forms a direct system of $P$-algebras and $A_{P}^{A}(R) \simeq \underset{\alpha}{\lim } A_{P}^{A}\left(R_{\alpha}\right)$.

8) Let $R=P\left[X_{\imath}\right]_{, \in \Gamma}$ be a polynomial ring over $P$ in indeterminates $X_{\text {, }}$, then we have $A_{P}^{A}(R) \simeq R\left[X_{1, a}\right]_{, \in r, a \in 1-\circ}$ a polynomial ring over $R$ in indeterminates $X_{\imath, a}$, where $d_{R / p}^{a} X_{\imath}$ corresponds to $X_{\iota, a}$ for every $\boldsymbol{a} \in 1-\boldsymbol{o}$ and $\iota \in \Gamma$.

9) If $R_{1}=R\left[X_{1}\right]_{1 \in \Gamma}$ is a polynomial ring over $R$ in indtemrinates $X_{1}$, then we have $A_{P}^{A}\left(R_{1}\right) \simeq A_{P}^{A}(R)\left[X_{a}\right]_{a \in r, a \in A}$ a polynomial ring over $A_{P}^{A}(R)$ in inaeterminates $X_{\iota, a}$, where $d_{R / P}^{a} X_{c}$ corresponds to $X_{\imath, a}$ for every $\iota \in \Gamma$ and $\boldsymbol{a} \in \Lambda$.

\section{§2. Differential algebras of some important algebras.}

Proposition 2. 1. Let $P$ be a ring with a linear topology. Let $R$ be a P-algebra with a topology induced by that of $P$. We consider a topology in $A_{P}^{A}(R)$ which is also induced by that of $P$. Then $R$ is a formally smooth $P$-algebra (Grothendieck [2]) if and only if $A_{P}^{A}(R)$ is a formally smooth $P$-algebra.

Proof. Let $E$ be a discrete $P$-algebra and let 5 be an ideal of $E$ such that $\mathbb{C}^{2}=(0)$. Let $\lambda: E \longrightarrow E / \subseteq$ be a canonical projection. First assume that $A_{P}^{A}(R)$ is a formally smooth $P$-algebra and a continuous $P$-algebra homomorphism $\bar{\varphi}: R \longrightarrow E / \mathbb{C}$ is given. Let $\mu: A_{P}^{A}(R) \longrightarrow A_{P}^{A}(R) / \bigoplus_{a \neq 0} A_{P}^{a}(R) \simeq R$ be a canonical projection, which is continuous. Then by the formal smoothness of $A_{P}^{A}(R)$, there exists a continuous $P$-algebra homomorphism $\bar{\varphi}: A_{P}^{A}(R) \longrightarrow$ $E$, satisfying the commutative diagram:

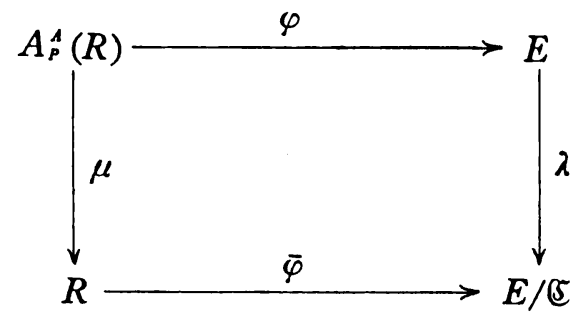


Since $\mu \circ d_{R / P}^{o}=i d_{R}$, we have $\lambda \circ\left(\varphi \circ d_{R / P}^{0}\right)=\bar{\varphi}$, which proves the formal smoothness of $R$. Next, assume that $R$ is a formally smooth $P$-algebra and a continuous $P$-algebra homomorphism $\bar{\psi}: A_{P}^{A}(R)$ $\longrightarrow E / \mathfrak{S}$ is given. Let $\mathfrak{\Im}_{A}$ and $\mathfrak{\Im}_{A}$ be ideals in the formal power series rings $E\left[\left[t_{1}, \ldots, t_{m}\right]\right]$ and $(E / \mathbb{E})\left[\left[t_{1}, \ldots, t_{m}\right]\right]$, respectively, generated by monomials $t^{\boldsymbol{a}} s$ with $\boldsymbol{a} \in \mathbf{N}_{0}^{m}, \boldsymbol{a} \notin \Lambda$. Let $\Omega$ be the kernel of the natural projection $\pi: E\left[\left[t_{1}, \ldots, t_{m}\right]\right] / \Im_{A} \longrightarrow$ $(E / \mathfrak{S})\left[\left[t_{1}, \ldots, t_{m}\right]\right] / \widetilde{\Im}_{\Lambda}$, where we regard both rings as discrete $P$ algebras. Then we have $\Omega^{2}=(0)$. We denote by $t_{i}^{\prime}$ the class of $t_{i}$ in both rings for each $i=1,2, \ldots, m$. We define a $P$-algebra homomorphism $\bar{g}: R \longrightarrow(E / \mathcal{C})\left[\left[t_{1}, \ldots, t_{m}\right]\right] / \widetilde{\Im}$ by $\bar{g}(x)=\sum_{a \in A} \bar{\psi} \circ d_{R / P}^{a}$ $(x) t^{\prime a}$ for $x \in R$. Since $\bar{\psi}$ is continuous, the $d_{R / P}^{a}$ are $P$-linear and $R$ and $A_{P}^{A}(R)$ have induced topologies, we see that $\bar{g}$ is continuous. Therefore, by our assumption there exists a continuous $P$-homomorphism $g: R \longrightarrow E\left[\left[t_{1}, \ldots, t_{m}\right]\right] / \Im_{A}$, which induces $\bar{g}$. Let $\partial=$ $\left\{\partial^{a}\right\}_{a \in \Lambda}$ be a higher $P$-derivation of $R$ into $E$ such that $g(x)=\sum_{a \in A}$ $\left(\partial^{a} x\right) t^{\prime a}$ with $\partial^{a} x \in E$ for $x \in R$. Then we have a $P$-algebra homomorphism $\phi: A_{P}^{A}(R) \longrightarrow E$ such that $\partial^{a}=\phi \circ d_{R / P}^{a}$ for every $\boldsymbol{a} \in \Lambda$. It is easily seen that $\phi$ induces $\bar{\phi}$. i Since $A_{P}^{A}(R)$ is generated by $d_{R / P}^{a}(R) \quad(\boldsymbol{a} \in \Lambda)$ and has an induced topology, we see that $\phi$ is continuous. This proves the formal smoothness of $A_{P}^{A}(R)$.

Proposition 2. 2. Let $R$ be a P-algebra. Let $S$ be a formally smooth $R$-algebra with respect to the discrete topology. Then the canonical $S$-algebra homomorphism $\lambda: S \otimes_{R} A_{P}^{A}(R) \longrightarrow A_{P}^{A}(S)$ is left inversible (,that is, there exists an $S$-algebra homomorphism $\mu: A_{P}^{A}(S) \longrightarrow S \otimes A_{P}^{A}$ (R) such that $\mu \circ \lambda=$ identity).

Proof. Let $\Im_{A}$ and $\widetilde{\Upsilon}_{h}(h=0,1,2, \ldots)$ be ideals of $S \otimes{ }_{R} A_{P}^{A}(R)$ such that

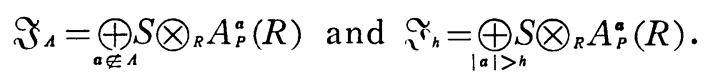

We put $T=S \otimes_{R} A_{P}^{A}(R) / \Im_{A}, \quad \overline{\mathfrak{J}}_{h}=\mathfrak{\Im}_{h}+\mathfrak{\Im}_{A} / \mathfrak{\Im}_{A}(h=0,1,2, \ldots)$ and 
$\tilde{T}=\underset{h}{\lim } T / \overleftarrow{\Im}_{h}$. If we introduce multiplications in $\underset{a \in A}{\bigoplus_{a}} S \bigotimes_{R} A_{P}^{a}(R)$ and in $\prod_{a \in A} S \otimes_{R} A_{P}^{a}(R)$, in the understandable way, we have

$$
T \simeq \bigoplus_{a \in A} S \otimes_{R} A_{P}^{a}(R) \text { and } \tilde{T} \simeq \prod_{a \in A} S \otimes_{R} A_{P}^{a}(R) .
$$

$\tilde{T}$ has an $R$-algebra structure $\psi$ defined by $\phi(x)=\left(1 \otimes d_{R / P}^{a} x\right)_{a \in \Lambda}$, $x \in R$ and we consider an $R$-algebra structure for $T / \Im_{h}$ induced by $\phi$ for every $h=0,1,2, \ldots$ Since we have an $R$-algebra isomorphism $T / \mathfrak{\Im}_{0} \simeq S$ and $S$ is a formally smooth $R$-algebra, the identical map $\varphi_{0}: S \longrightarrow S \simeq T / \widetilde{\Im}_{0}$ can be successively lifted to $R$ algebra homomorphisms $\varphi_{h}: S \longrightarrow T / \mathfrak{\Im}_{h}(h=0,1,2, \ldots)$. Take the projective limit $\varphi: S \longrightarrow \tilde{T}$ of $\varphi_{h}$. Then we have a commutative diagram :

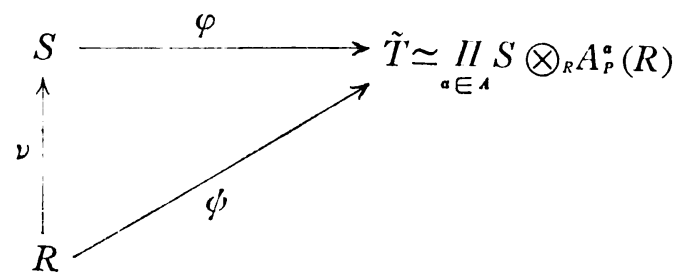

, where $\nu$ is the structure homomorphism of the $R$-algebra $S$. Give the co-ordinate wise expression of $\varphi, \varphi(x)=\left(\partial^{a} x\right)_{a \in A}, x \in S$. Then $\partial=\left\{\partial^{a}\right\}_{a \in A}$ is a higher $P$-derivation of $S$ into $S \otimes_{R} A_{P}^{A}(R)$ with index domain $A$ and we have commutative diagrams

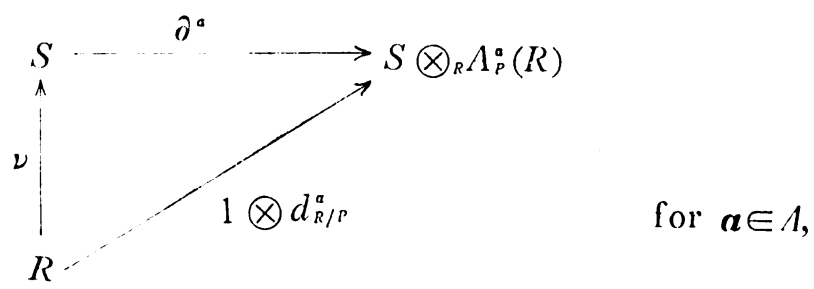

and $\partial^{\circ}$ is the structure homomorphism of the $S$-algebra $S \otimes_{R} A_{P}^{A}(R)$. Therefore, if we denote by $\mu$ the $S$-algebra homomorphism of 
$A_{P}^{A}(S)$ into $S \otimes_{R} A_{P}^{A}(R)$ such that $\hat{o}^{a}=\mu \circ d_{s / P}^{a}$ for $\boldsymbol{a} \in \Lambda$, we have a commutative diagram:

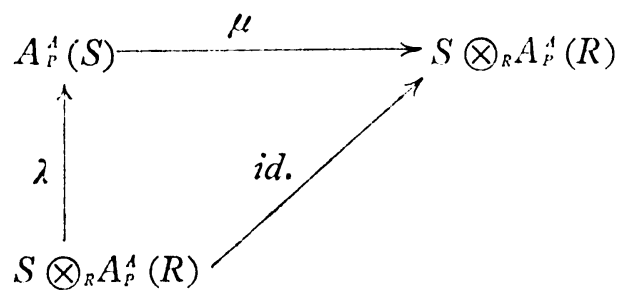

, which proves our proposition.

Corollary. Let $k$ be a field. Let $K$ be a field containing $k$. Let $L$ be a field which is a separable extension of $K$. Then the canonical homomorphism ;

$$
\mu: L \otimes{ }_{k} A_{k}^{A}(K) \longrightarrow A_{k}^{A}(L)
$$

is left inversible.

Proof. Since $L$ is a formally smooth $K$-algebra (Grothendieck [2]), our assertion is a direct consequence of Proposition 2.2.

Proposition 2. 3. Let $P$ be a ring with a linear topology. Let $R$ be a P-algebra with a topology induced by that of $P$. Assume that $R$ is a formally unramified P-algebra (Grothendieck [2]). Then, for every open ideal $\mathfrak{A}$ of $R$ and $\boldsymbol{a} \in 1-\boldsymbol{o}$ it holds that

$$
A_{P}^{\mathrm{a}}(R) \subset \mathfrak{N} A_{P}^{\mathbf{a}}(R)
$$

and we have a canonical isomorphism :

$$
A_{P}^{A}(R) / \mathfrak{Y} A_{P}^{A}(R) \simeq R / \mathfrak{P}
$$

Proof. The last assertion is an immediate consequence of the first assertion. To prove the first assertion, it is enough to prove that for every $\boldsymbol{a} \in \Lambda-\boldsymbol{o}$ we have $d_{R / P}^{a} R \subset \mathfrak{Y} A_{P}^{a}(R)$, because $A_{\boldsymbol{p}}^{A}(R)$ 
is generated by the $d_{R / P}^{a} R$. Assume that there exists an $\boldsymbol{a} \in \Lambda_{-0}$ such that $d_{R / P}^{a} R \not \subset \mathfrak{A} A_{P}^{a}(R)$. We may assume that $|\boldsymbol{a}|=h$ is the least integer with this property. Let $\Im$ and $\Re$ be two ideals of $A_{P}^{A}(R)$ such that $\mathfrak{\Im}=\sum_{|a| \geq h} A_{P}^{a}(R)$ and $\mathfrak{R}=\sum_{|a|\rangle_{h}} A_{P}^{a}(R)$. We put

$$
E=A_{P}^{A}(R) / \mathfrak{A} A_{P}^{A}(R)+\Re \text { and } \mathfrak{C}=\mathfrak{S}+\mathfrak{A} A_{P}^{A}(R) / \mathfrak{A} A_{P}^{A}(R)+\Re .
$$

Then we have $\mathfrak{C}^{2}=0$ and $E / \mathfrak{C} \simeq R / \mathfrak{A}$. Let $\varphi$ and $\phi$ be two $P$ algebra homomorphisms of $R$ into $E$;

$$
\varphi=d_{R / P}^{o} \quad \bmod . \mathfrak{H} A_{P}^{A}(R)+\Re, \Psi=\sum_{|a| \leq h} d_{R / P}^{a} \quad \bmod . \mathfrak{H} A_{P}^{A}(R)+\Re .
$$

Since $R$ has an induced topology, it is easy to see that $\varphi$ and $\phi$ be continuous when we regard $E$ as a discrete $P$-algebra. On the other hand, $\phi \neq \varphi$ by our assumption and both induce modulo C the canonical surjection $R \longrightarrow R / \mathfrak{A}$. This contradicts the formal unramifiedness of $R$ over $P$.

Corollary. Let $R$ be a formally unramified $P$-algebra with respect to the discrete topology. Then we have $A_{p}^{A}(R)=A_{p}^{\circ}(R) \simeq R$.

Proposition 2. 4. Let $R$ be a P-algebra. Let $S$ be a formally étale $R$-algebra with respect to the discrete topology. Then we have a canonical isomorphism :

$$
S \otimes_{R} A_{P}^{A}(R) \simeq A_{P}^{A}(S)
$$

Proof. By Proposition 2. 2, $S \bigotimes_{R} A_{P}^{A}(R) \longrightarrow A_{P}^{A}(S)$ is left inversible. By the corollary to Proposition 2. 3, we have $A_{R}^{A}(S) \simeq S$. Therefore our assertion follows from Proposition 1.1, 5).

Corollary 1. Let $\gamma$ be a multiplicatively closed subset of $R$. Then we have a canonical isomorphism :

$$
R \gamma \otimes_{R} A_{P}^{A}(R) \simeq A_{P}^{A}(R \gamma)
$$


Proof. This follows from Proposition 2.4, because $R \gamma$ is a formally étale $R$-algebra (Grothendieck [2]).

Corollary 2. Let $k$ be a field. Let $K$ be a field containing $k$. Let $L$ be a separably algebraic extension of $K$. Then we have a canonical isomorphism :

$$
L \bigotimes_{K} A_{k}^{A}(K) \simeq A_{k}^{A}(L)
$$

Proof. If $L$ is a finite extension of $K, L$ is a formally étale $K$-algebra (Grothendieck [2]). Hence our assertion follows from Proposition 2. 4 and Proposition 1.1,7).

\section{§3. Differential algebras of regular local rings.}

For the proof of the following proposition, we refer to Berger [1] or Kawahara-Yokoyama [6], although for the case of characteristic 0 , it is an immediate consequence of Proposition 1. 1, 8), and Corollary 1 and 2 to Proposition 2. 4.

Proposition 3. 1. Let $K$ be a field. Let $\left\{c_{c}\right\}_{, \in r}$ be a transcendence base of $K$ in case the characteristic of $K$ is 0 and a $p$ independent base in case the characteristic of $K$ is $p \neq 0$. Then we have

$$
A^{A}(K)=K\left[d^{a} c_{\imath}\right]_{a \in A-0, \imath \in \Gamma}
$$

, where the right hand side is a polynomial ring over $K$ in distinct indeterminates $d^{a} c_{\imath}$.

Let $R$ be a local $P$-algebra. Let $\|$ be an ideal of $R$. We denote by the symbol the ut-adic completions of $R$-algebras.

Proposition 3. 2. We have a canonical isomorphism :

$$
\widehat{A_{P}^{A}(R)} \simeq \widehat{A_{P}^{A}(\hat{R})}
$$


Proof. Let $i: R \longrightarrow R$ be the canonical embedding. $i$ induces a canonical homomorphism of $A_{P}^{A}(R)$ to $A_{P}^{A}(R)$, which is extended to

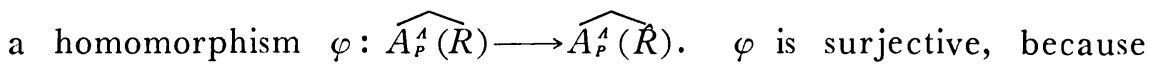
$A_{P}^{A}(R)$ and $A_{P}^{A}(\hat{R})$ are generated by $d_{R / P}^{a} R$ and $d_{R / P}^{a} \hat{R}$. $\quad(\boldsymbol{a} \in \Lambda)$, respectively, and the $d_{R / P}^{a}$ are continuous (in 11 -adic topology). By the continuity of the $d_{R / P}^{a}$, they are extended to a higher derivation of $\hat{R}$ into $\widehat{A_{P}^{A}(R)}$. Hence we get a homomorphism of $A_{P}^{A}(\hat{R})$ into

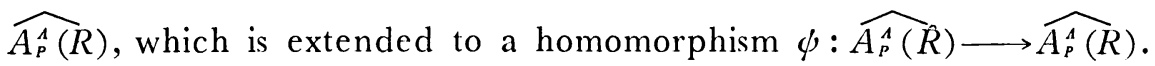
It is easy to see that $\phi \circ \varphi=$ identity.

Lemma 3. 1. Let $A$ and $B$ be $R$-algebras, such that

1) $A$ is a formally smooth $R$-algebra with respect to $\mathrm{m}$-adic topology,

2) $B / \mathrm{m}^{n} B$ is $R / \mathrm{m}^{n}$-flat for all $n>0$ and

3) there is an $R$-algebra isomorphism $f_{1}: A / m A \longrightarrow B / m B$. Then the following assertions $a), b$ ) and $c$ ) hold.

a) There exist $R$-algebra isomorphisms $f_{n}: A / \mathrm{m}^{n} A \longrightarrow B / \mathrm{m}^{n} B$ for $n=1,2, \ldots$, making the following diagram commutative:

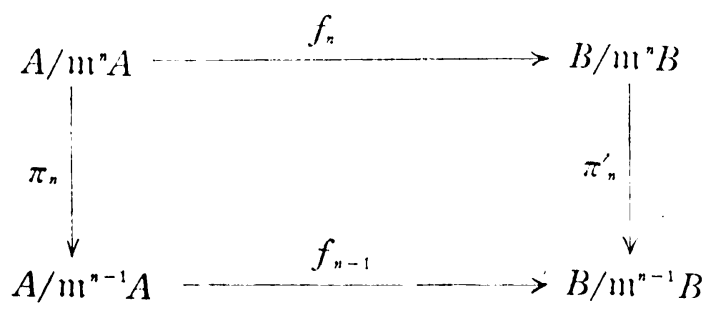

, where the $\pi_{n}$ and the $\pi^{\prime}{ }_{n}$ are natural projections.

b) We have an $\hat{R}$-algebra isomorphism $\hat{A} \simeq \hat{B}$.

c) Every $R$-algebra homomorphism of $\hat{A}$ into $\hat{B}$ which induces $f_{1}$, is an isomorphism.

Proof. b) follows immediately from a). We prove a) by induction on $n$. Assume that we obtain $f_{1}, \ldots, f_{n-1}$ with the said property. Let $\gamma_{n}: A \longrightarrow A / \mathrm{m}^{n} A$ be a natural projection. Then 
by the formal smoothness of $A$ there exists an $R$-algebra homomorphism $g_{n}: A \longrightarrow B / \mathrm{m}^{n} B$ satisfying the commutative diagram:

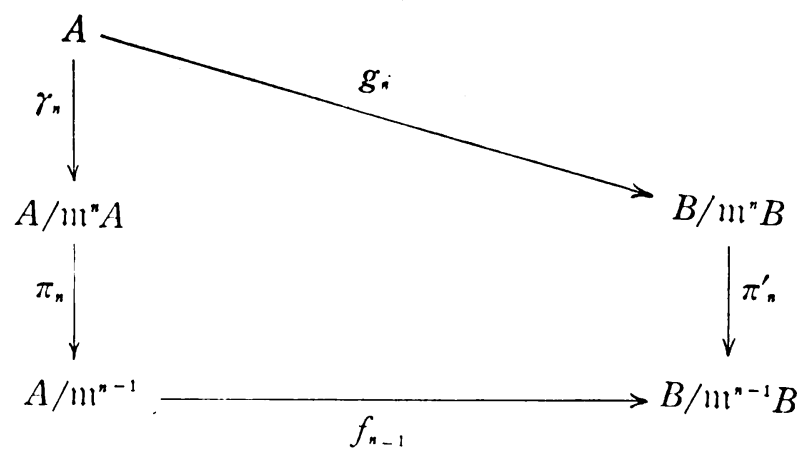

$g_{n}$ induces an $R$-algebra homomorphism $f_{n}: A / \mathrm{m}^{n} A \longrightarrow B / \mathrm{m}^{n} B$, which satisfies the commutative diagram in a). The surjectivity of $f_{n}$ is clear. Assume that $0 \longrightarrow K \longrightarrow A / \mathfrak{m}^{n} A \stackrel{f_{n}}{\longrightarrow} B / \mathfrak{m}^{n} B \longrightarrow 0$ is an exact sequence of $R / m^{n}$-modules. Then we have an exact sequence :

$$
\begin{aligned}
\ldots \longrightarrow \operatorname{Tor}_{1}^{R / m^{n}}\left(B / \mathrm{m}^{n}, R / \mathrm{m}^{n-1}\right) \longrightarrow & K \bigotimes_{R / \mathrm{m}^{n}} R / \mathrm{m}^{n-1} \longrightarrow A / \mathrm{m}^{n-1} A \longrightarrow \\
\longrightarrow B / \mathrm{m}^{n-1} B \longrightarrow 0 &
\end{aligned}
$$

Since $B / \mathrm{m}^{n}$ is $R / \mathrm{m}^{n}$-flat, we have $\operatorname{Tor}_{1}^{R / \mathrm{m}^{n}}\left(B / \mathrm{m}^{n}, R / \mathrm{m}^{n-1}\right)=0$. Therefore it holds that $K \bigotimes_{R / m^{n}} R / \mathfrak{m}^{n-1}=0$, hence we have $K=0$ by Nakayama's lemma for nilpotent ideals. Hence we have $A / \mathrm{m}^{n} A$ $\simeq B / \mathfrak{m}^{n} B$. The assertion $\mathrm{c}$ ) is clear from the above argument. $f_{n}$

Proposition 3. 3. Let $P$ be a discrete valuation ring with a prime element $p$, where $p$ is a prime integer. Let $\left\{c_{c}\right\}_{\llcorner\in \Gamma}$ be a set of representatives of a p-independent base of $P / p P . \quad$ Let $Z_{\iota, a}(\iota \in \Gamma, \boldsymbol{a} \in \Lambda-\boldsymbol{o})$ be indeterminates. Then we have a P-algebra isomorphism:

$$
\widehat{A^{A}(P)} \simeq \widehat{P\left[Z_{\imath, a}\right]}, \in r_{, a \in A-。}
$$

, where the symbol $\widehat{ }$ denotes $p$-adic completions and the $d_{P}^{a} c_{1}$ are mapped to the $Z_{\imath, a}$. 
Proof. We denote by the $\bar{c}_{\text {, the }}$ classes of the $c$, modulo $p P$ and by the $\bar{Z}_{\imath, a}$ those of the $Z_{\imath, a}$ modulo $p P\left[Z_{\imath, a}\right]$. Then by Proposition 3.1 we have a $P$-algebra isomorphism $: A^{A}(P / p P) \simeq$ $P\left[Z_{\iota, a}\right] / p P\left[Z_{\imath, a}\right]$, where the $d_{P}^{a} c_{\iota}$ correspond to the $\bar{Z}_{\iota, a}(\iota \in \Gamma$, $\boldsymbol{a} \in 1-\boldsymbol{o})$. On the other hand, since $d_{P}^{a} p=0$ for every $\boldsymbol{a} \in \Lambda_{-\boldsymbol{o}}$, it holds that $A^{4}(P / p P) \simeq A^{4}(P) / p A^{4}(P)$ by Proposition 1.1,4). Hence we have an isomorphism $\bar{\varphi}: A^{A}(P) / p A^{A}(P) \stackrel{\sim}{\longrightarrow}\left[Z_{c, a}\right] / p P\left[Z_{\imath, a}\right]$. Since $P$ is a formally smooth $\mathbf{Z}_{p}$-algebra, $A^{A}(P)$ is a formally smooth $\mathbf{Z}_{p}$ algebra by Proposition 2. 1. Since $P$ is a Cohen ring, $P$ is a flat $\mathbf{Z}_{p}$-algebra (Grothendieck [2]). Hence, $P\left[Z_{t, a}\right] / p^{n} P\left[Z_{t, a}\right]$ is a flat $\mathbf{Z}_{p} / p^{n} \mathbf{Z}_{p}$-algebra for every $n>0$. Therefore by the above lemma, there exists a $\mathbf{Z}_{p}$-algebra isomorphism $\hat{\varphi}: \widehat{A^{A}(P)} \stackrel{\sim}{\sim} \widehat{P\left[Z_{\iota, a}\right]_{\iota \in \Gamma, a \in \Lambda-o}}$, On the other hand, we have a $P$-algebra homomorphism $\phi: P\left[Z_{\iota, a}\right] \longrightarrow A^{A}(P)$ such that $\phi\left(Z_{\iota, a}\right)=d_{p}^{a} c$, for $\iota \in \Gamma, \boldsymbol{a} \in \Lambda-\boldsymbol{o}$, which induces a homomorphism $\hat{\psi}: \widehat{P\left[Z_{1, a}\right]} \longrightarrow A^{A}(P)$. Since $\hat{\psi}$ induces the isomorphism $\bar{\varphi}^{-1}, \hat{\psi}$ is surjective and $\hat{\varphi} \circ \hat{\phi}$ induces the identical isomorphism of $P\left[Z_{c, a}\right] / p P\left[Z_{\iota, a}\right]$. We apply again the above lemma on $\hat{\varphi} \circ \hat{\psi}$ and get that $\hat{\varphi} \circ \hat{\psi}$ is a $\mathbf{Z}_{p}$-algebra isomorphism. Hence $\hat{\psi}$ is an isomorphism.

Theorem 3. 1. Let $R$ be an unramified regular local ring with maximal ideal $11 \mathrm{t}$. Let

1) $x_{1}, \ldots, x_{n}$ be a regular system of parameters in case $R$ is of equal characteristic, and

2) $p, x_{1}, \ldots, x_{n}$ be a regular system of parameters in case $R$ is of unequal characteristic, where $p$ is the characteristic of $R / 11$.

Let $\left\{c_{c}\right\}_{\iota \in \Gamma}$ be a set of elements in $R$ such that the set $\left\{\bar{c}_{\iota}\right\}_{\iota \in r}$ of their residue classes modulo $\mathrm{nt}$ are

a) a transcendence base of $R / \mathrm{m}$ in case the characteristic of $R / \mathrm{m}$ is 0 , and

b) a p-independent base of $R / m$ in case the characteristic of $R / \mathrm{m}$ is $p \neq 0$. 
Then we have:

$$
\widehat{A^{A}(R)}=R \widehat{\left[d^{a} c_{\imath}, d^{b} x_{i}\right]}{ }_{\imath \in r, 1 \leq i \leq n, a, b \in A-o}
$$

, where the latter is a completion of a polynomial ring over $R$ in distinct indeterminates $d^{a} c_{\imath}$ 's and $d^{b} x_{i}{ }^{\prime} s$, and the symbol denotes the $111-$ adic completions.

Proof. This is a consequence of Proposition 3.1, Proposition 3. 2, Proposition 3.3 and Proposition 1. 1,8).

Corollary. We retain notations and assumptions as in Theorem 3.1. Let $S$ be an R-algebra and complete in nt-adic topology. A higher derivation $\partial=\left\{\hat{\partial}^{a}\right\}_{a \in \Lambda}$ of $R$ into $S$ with index domain $A$, where $\partial^{\circ}$ is the structure homomorphism of $S$, is uniquely determined assigning values of $\partial^{a} c_{\iota}, \partial^{b} x_{i}(\iota \in \Gamma, 1 \leq i \leq n, \boldsymbol{a}, \boldsymbol{b} \in \Lambda-\boldsymbol{o})$ arbitarily in $S$.

Proof. Since every $R$-algebra homomorphism of $A^{A}(R)$ into $S$ is uniquely extended to an $R$-algebra homomorbhism of $\widehat{A^{A}(R)}$ into $S$, our assertion follows from Theorem 3.1 and Proposition 1.2.

\section{$\S 4$. Differential algebras of complete discrete valuation rings.}

We list below some of notations and conventions which are common in $\S 4, \S 5, \S 6$ and $\S 7$.

$R$ : a complete discrete valuation ring of unequal characteristic.

III: the maximal ideal of $R$.

$\pi$ : a prime element of $R$.

$v$ : the valuation of $R$.

$k$ : the residue field of $R$.

$p:$ the characteristic of $K$.

$e$ : the ramification index of $R$.

$P$ : a coefficient ring of $R$.

$\left\{\bar{c}_{c}\right\}_{\iota \in \Gamma}:$ a $p$-independent base of $k$.

$c_{\iota}(\iota \in \Gamma):$ a representative of $\bar{c}_{\iota}$ contained in $P$. 
$S$ : the polynomial ring $P[X]$ in an indeterminate $X$ over $P$. $f(X)=X^{\bullet}+p\left(a_{\bullet_{-1}} X^{\bullet-1}+\ldots+a_{0}\right):$ the Eisenstein polynomial over $P$ such that $f(\pi)=0$.

$\Lambda$ : a segment $\{0,1,2, \ldots, l\}$ of $\mathbf{N}_{0}$, where $l$ may be $\infty$ in which case $\Lambda=\mathbf{N}_{0}$.

: a symbol for the $p$-adic completion of a $P$-algebra. (If the $P$-algebra is considered as an $R$-algebra, $\widehat{T}$ represents also the m-adic completion.)

$\varphi$ : the canonical $R$-algebra homomorphism: $R \otimes_{P} A^{A}(P) \longrightarrow$ $A^{A}(R)$.

$\hat{\varphi}$ : the canonical $R$-algebra homomorphism $: \widehat{R \otimes_{P} A^{A}(P)} \longrightarrow$ $\widehat{A^{A}(R)}$.

$d^{i} x \quad(i \in \Lambda, x \in S)$ : the canonical image of $d_{s}^{i} x \quad\left(\in A^{A}(S)\right)$ in $R \otimes_{s} A^{4}(S)$ or in $\widehat{R \otimes_{s} A^{4}(S)}$.

$$
\left.\begin{array}{l}
Z_{i}, U_{h, j}(i \geq 1,0 \leq e-1, j \geq 1): \\
Z_{i}, W_{t, j}(i \geq 1, \iota \in \Gamma, i \geq 1):
\end{array}\right\} \text { independent variables over } R .
$$

We assign weight $Z_{i}=$ weight $W_{\imath, i}=i$ for every $\iota \in \Gamma, i=1,2, \ldots$.

When we say that a grade preserving $R$-algebra homomorphism $\rho$ of a graded $R$-algebra $H$ into a graded $R$-algebra $K$, is left inversible, we mean that there exists a grade preserving $\mathrm{R}$-algebra homomorphism $\omega$ of $K$ into $H$ such that $\omega \circ \rho=i d_{H}$.

By Proposition 1.1,8), $A_{P}^{A}(S)$ is a polynomial ring $S\left[d_{s / P}^{i} X\right]_{i \in 1-o}$ in distinct indeterminates $d_{s / P}^{i} X(i \in \Lambda-0)$. We have a formula for every $n=1,2, \ldots$ (Ribenboim [8]) :

$$
d_{s / P}^{n} f(X)=\sum_{j=1} \frac{f^{(j)}(X)}{j !} \sum_{\substack{i_{1}+\cdots+i_{j}=n \\ 1 \leq i_{1}, \cdots, i_{j}}} d_{s / P}^{i_{1}} X \ldots d_{s / P}^{i_{j}} X
$$

, which follows from the fact:

$$
\begin{aligned}
d_{s / P}^{n} X^{m} & =\sum_{\substack{i_{1}+\cdots,+i_{m}=n \\
0 \leq i_{1}, \cdots, i_{m}}} d_{s / P}^{i_{1}} X \ldots d_{s / P}^{i_{m}} X \\
& =\sum_{j=1}^{m}\left(\begin{array}{c}
m \\
m-j
\end{array}\right) X^{m-j} \sum_{\substack{i_{1}+\cdots, i_{j=n} \\
1 \leq i_{1}, \cdots, i_{j}}} d_{s / P}^{i_{1}} X \ldots d_{s / P}^{i_{j}} X
\end{aligned}
$$




$$
\begin{array}{r}
=\sum_{j=1}^{m} \frac{\left(X^{m}\right)^{(j)}}{j !} \sum_{\substack{i_{1}+\cdots+i_{j}=n \\
1 \leq i_{1}, \cdots i j}} d_{s \mid p / p}^{i_{1}} X \ldots d_{s / p}^{i_{j}} X \text { for every } \\
m=1,2, \ldots .
\end{array}
$$

By a slight modification of the above proof, we can obtain a relation for every $n=1,2, \ldots$ :

$$
\begin{array}{r}
d_{s}^{n} f(X)=\sum_{j=1} f_{j !}^{(j)}(X) \sum_{\substack{i_{1}+\cdots,+i_{j}=n \\
1 \leq i_{1}, \ldots, i_{j}}} d_{s}^{i_{1}} X \ldots d_{s}^{i_{j}} X+ \\
p G_{n}\left(d_{s}^{1} X, d_{s}^{2} X, \ldots ; d_{s}^{1} a_{0}, d_{s}^{2} a_{0}, \ldots, d_{s}^{1} a_{\bullet-1}, d_{s}^{2} a_{e-1}, \ldots\right)
\end{array}
$$

, where $G_{n}\left(Z_{1}, Z_{2}, \ldots, U_{0,1}, U_{0,2}, \ldots, U_{e-1,1}, U_{\epsilon-1,2}, \ldots\right)$ is a polynomial of the same weight $n$ and has no terms of monomials of only $Z_{i}^{\prime} s$.

Since $R \simeq S /(f(X))$ and $A^{A}(S) \simeq A^{A}(P)\left[d_{s}^{i} X\right]_{i \in A}$ $\simeq\left(P[X] \otimes_{P} A^{A}(P)\right)\left[d_{s}^{i} X\right]_{i \in A-0}$, we have :

$$
A^{A}(R) \simeq\left(R \otimes_{P} A^{A}(P)\right)\left[d^{i} X\right]_{i \in A-o} /\left(d^{n} f(X)\right)_{n \in A-o}
$$

, where $\left(R \otimes_{P} A^{A}(P)\right)\left[d^{i} X\right]_{i \in A-\circ}$ is a polynomial ring over $R \otimes_{P} A^{A}$ $(P)$ in $\left\{d^{i} X\right\}_{i \in A-o}$.

Take nt-adic completions of both hand sides and we have by Proposition 3. 3:

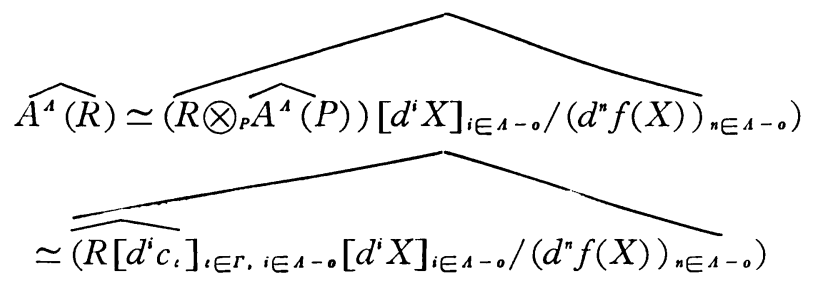

, where $R\left[d^{i} c_{\imath}\right]_{\iota \in \Gamma, i \in A_{-} \circ}$ is a polynomial ring over $R$ in $\left\{d^{i} c_{\imath}\right\}_{\imath \in \Gamma, i \in A-o}$

Expressing the $d^{i} a_{j}$ by the $d^{i} c_{\imath}$, we get a relation in $\widehat{R \otimes_{s} A^{4}}(S)$ for every $n \in A-o$ from (2):

$$
\begin{aligned}
d^{n} f(X)= & \dot{j} \frac{f^{(j)}(\pi)}{j !} \sum_{\substack{i_{1}+\cdots+j_{i}=n \\
1 \leq i_{1}, \cdots i_{j}}} d^{i_{1}} X \ldots d^{i j} X+ \\
& +p \bar{G}_{n}\left(d^{1} X, d^{2} X, \ldots ; \ldots, d^{i} c_{\imath}, \ldots\right)
\end{aligned}
$$

, where (i) $\bar{G}_{n}\left(Z_{1}, Z_{2}, \ldots ; \ldots, W_{\iota, i}, \ldots\right)$ is a linear combination 
of monomials of $Z_{i}^{\prime} s$ and $W_{\imath, i}$ 's of the same weight $n$ with coefficients in $R$, (ii) for every positive integer $t$ all but a finite number of coefficients of $\bar{G}_{n}$ are in $\mathrm{m}^{t}$ and (iii) $\bar{G}_{n}$ has no terms of monomials of only $Z_{i}^{\prime} s$.

Proposition 4. 1. A higher derivation $\partial=\left\{\partial^{n}\right\}_{n \in A}$ of $R$ into an u-adic complete $R$-algebra $T$ with index domain $\Lambda$ such that $\partial^{0}=$ the structure homomorphism of $T$, is uniquely determined by assigning values of $\partial^{i} c$, and $\partial^{i} \pi(\iota \in \Gamma, i \in \Lambda-\mathbf{0})$ in $T$, satisfying conditions :

$$
\sum_{j=1}^{\dot{1}} \frac{f^{(j)}}{j !} \sum_{\substack{i_{1}+\cdots,+i_{j}=n \\ i_{\leq 1}, \cdots, i_{j}}} \partial^{i_{1}} \pi \ldots \partial^{i_{j}} \pi+p \bar{G}_{n}\left(\partial^{1} \pi, \partial^{2} \pi, \ldots ; \ldots \partial^{i} c_{\imath} \ldots\right)=0
$$

for all $n=1,2, \ldots, l$.

Proof. By the condition that $\partial^{0}=$ the structure homomorphism of $T, \partial$ is uniquely determined by an $R$-algebra homomorphism $g$ : $A^{A}(R) \longrightarrow T$. Since $T$ is complete, $g$ is uniquely extended to an $R$-algebra homomorphism $\hat{g}: \widehat{A^{A}(R)} \longrightarrow T$. Hence our assertion follows from the above argument.

\section{§5. Extended Neggers' numbers $\Delta_{P}^{n}(\pi)$.}

Multiplying $\left(f^{\prime}(\pi)\right)^{2 n-2}$ on both hand sides of (3), we obtain a relation for each $n \in \Lambda-o$ :

$$
\begin{gathered}
(\overline{3}) \quad\left(f^{\prime}(\pi)\right)^{2 n-2} d^{n} f(X)=\left(f^{\prime}(\pi)\right)^{2 n-1} d^{n} X+\sum_{j=2} \frac{f^{(j)}(\pi)}{j !}\left(f^{\prime}(\pi)\right)^{j-2} \\
\sum_{\substack{i_{1}+\cdots, i_{j}=n \\
1 \leq i_{1}, \ldots, i_{j}}}\left(f^{\prime}(\pi)\right)^{2 i_{1}-1} d^{i_{1}} X \ldots\left(f^{\prime}(\pi)\right)^{2 i_{j}-1} d^{i_{j}} X+p H_{n}\left(f^{\prime}(\pi) d^{1} X,\right. \\
\left.\left(f^{\prime}(\pi)\right)^{3} d^{2} X, \ldots ; \ldots, d^{i} c_{\iota}, \ldots\right)
\end{gathered}
$$

, where $H_{n}\left(f^{\prime}(\pi) d^{1} X, \ldots ; \ldots, d^{i} c_{\imath}, \ldots\right)=\left(f^{\prime}(\pi)\right)^{2 n-2} G_{n}\left(d^{1} X, \ldots ; \ldots, d^{i} c_{\imath}, \ldots\right)$.

Letting the left hand side of this relation equal 0 , we can successively solve $\left(f^{\prime}(\pi)\right)^{2 n-1} d^{n} X$ and obtain the following relation in $\widehat{A^{A}(R)}$ for $n \in \Lambda-o$, where we denote the canonical images 
of the $d_{R}^{i} X\left(i \in \Lambda^{-o}, x \in R\right)$ in $\widehat{A^{4}(R)}$ by the same symbols.

$$
\left(f^{\prime}(\pi)\right)^{2 n-1} d_{R}^{n} \pi=F_{n}\left(\ldots, d_{R}^{i} c_{1}, \ldots\right)
$$

, where $F_{n}\left(\ldots, W_{\imath, i}, \ldots\right)$ is an $R$-linear combination of countably many monomials of the $W_{\iota, i}(\iota \in \Gamma, 1 \leq i \leq n)$ of the same weight n. Then from Proposition 4.1 and the fact that $R$ is an integral domain and $f^{\prime}(\pi) \neq 0$, we can easily deduce the following.

Proposition 5. 1. A higher derivation $\partial=\left\{\partial^{n}\right\}_{n \in A}$ of $R$ into $R$ with index domain $\Lambda$ such that $\hat{o}^{0}=$ the structure homomorphism, is uniquely determined by assigning values of $\partial^{i} c$, and $\partial^{i} \pi(\iota \ni \Gamma, i \in \Lambda-\boldsymbol{o})$ in $R$, satisfying conditions

$$
\left(f^{\prime}(\pi)\right)^{2 n-1} \partial^{n} \pi=F_{n}\left(\ldots, \partial^{i} c_{\iota}, \ldots\right) \text { for all } n=1,2, \ldots, l .
$$

Definition 5. 1. We define:

$$
J_{P}^{n}(\pi)=\min v\left(\text { coefficient of } F_{n}\right)-(2 n-1) v\left(f^{\prime}(\pi)\right)
$$

for $n=1,2, \ldots$, where we understand that

$$
\min v \text { (coefficeint of } F_{n} \text { ) }=\infty \text { if } F_{n}=0 \text {. }
$$

We call $\Delta_{P}^{n}(\pi)$ then-th Neggers' number for $(P, \pi)$.

Remark 5. 1. It is easy to see that $J_{P}^{1}(\pi)$ is the Neggers' number defined in Suzuki [10], which originally appeared in Neggers [7].

Remark 5. 2. If $k$ is perfect, $\Gamma$ is empty, hence $J_{P}^{n}(\pi)=\infty$ for $n=1,2, \ldots$.

Proposition 5. 2. $J_{p}^{n}(\pi)$ is independent of the choice of $\left\{c_{c}\right\}_{\iota \in r}$ for each $n=1,2, \ldots$

To prove Proposition 5.2, it is enough to give an alternative definition of $\Delta_{P}^{n}(\pi)$ without using $\left\{c_{t}\right\}_{\iota \in \Gamma}$, which is given in the following proposition. 
Proposition 5. 3. Let $n$ be a positive integer. Then $J_{p}^{n}(\pi)=h$ if and only if for a sufficiently large integer $t$ it holds that, for every higher derivation $\partial=\left\{\partial^{0}=i d_{R}, \partial^{1}, \ldots, \partial^{n}\right\}$ of $R$ into $R$ with index domain $\{0,1, \ldots, n\}$ such that $v\left(\partial^{i} b\right) \geq t i$ for all $b \in P$ and $0<i \leq n$, we have $v\left(\partial^{n} \pi\right) \geq t n+h$ and there exists a higher derivation $\partial$ with this property such that $\min v\left(\hat{\sigma}^{n} \pi\right)=t n+h$.

Proof. If $k$ is perfect, our assertion follows from Remark 5.2 and Proposition 5.1. Hence we may assume that $k$ is an infinite field. We put $s=\min v$ (coefficient of $F_{n}$ ). Let $t$ be a fixed positive integer. If $w_{\imath, \mathrm{i}}(\iota \in \Gamma, i=1,2, \ldots, n)$ are elements in $R$ such that $v\left(w_{c, i}\right) \geq t i$, we have $v\left(F_{n}\left(\ldots, w_{c, i}, \ldots\right)\right) \geq s+t n$. As it will be shown in the lemma below we can choose $w_{c, i}$ 's such that $v\left(F_{n}\left(\ldots, w_{1, i}, \ldots\right)\right)=s+t n$. We choose $\mathrm{t}$ sufficiently large so that $J_{P}^{j}(\pi)+t j \geq 0$ for every $j=1,2, \ldots, n$. Then each of equations for the $\partial^{j} \pi$

$$
f^{\prime}(\pi)^{2 j-1} \partial^{j} \pi=F_{j}\left(\ldots, w_{\iota, i}, \ldots\right) \quad(j=1,2, \ldots, n)
$$

can be solved in $R$. Our assertion follows from these arguments easily.

Lemma 5. 1. Assume that $k$ is an infinite field. Let $F(\ldots$, $\left.W_{1, .} ..\right)$ be a linear combination of countably many monomials of $W_{\imath, i}$ of the same weight $n$ with coefflicients in $R$ such that for every positive integer $r$, all but a finite number of coefficients of $F$ are in $\mathrm{m}^{\prime}$. We put $s=\min v$ (coefficient of $F$ ). Let $t$ be a non-negative integer. Then, there exists a set of elements $\left\{w_{1, i}\right\}_{, \in r, i=1,2, \ldots}$ in $R$ such that $v\left(w_{\iota, i}\right) \geq t i$ for $\iota \in I$ and $i=1,2, \ldots$, and $v\left(F\left(\ldots, w_{\iota, i}, \ldots\right)\right)=s+t n$. Moreover, if $t=0$, we may choose the $w_{\imath, i}$ from $P$.

Proof. We may assume that $s \neq \infty$. Let $H\left(\ldots, W_{\iota, i}, \ldots\right)$ be a polynomial consisting of all terms of $F\left(\ldots, W_{c, i}, \ldots\right)$ such that the values of their coefficients under $v$ equal $s$. Then we can write 
as $H\left(\ldots, W_{\iota, i}, \ldots\right)=\pi^{s} H_{0}\left(\ldots, W_{\iota, i}, \ldots\right)$, where $H_{0}$ is a non-zero polynomial in the $W_{\iota, i}$ such that all non-zero coefficients of $H_{0}$ are units in $R$. Let $\bar{H}_{0}$ be a polynomial over $k$, induced by $H_{0}$ modulo nt. Then by our assumption, there exists a set of elements $\left\{\bar{u}_{\imath, i}\right\}$ $: \in r, i=1,2, \ldots$ in $k$ such that $\bar{H}_{0}\left(\ldots, \bar{u}_{\imath, i}, \ldots\right) \neq 0$. Then for an arbitrary set of representatives $\left\{u_{i, i}\right\}_{\iota \in r, i=1,2, \ldots}$ of $\left\{\bar{u}_{\iota, i}\right\}_{\iota \in r, i=1,2, \ldots}$ in $R, H_{0}\left(\ldots, u_{\imath, i}, \ldots\right)$ is a unit in $R$. We put $w_{\imath, i}=\pi^{i i} u_{\imath, i}$ for $\iota \in \Gamma, i=1,2, \ldots$. Then $v\left(H\left(\ldots, w_{\imath, i}, \ldots\right)\right)=v\left(\pi^{s+t n} H_{0}(\ldots\right.$, $\left.\left.u_{i, i}, \ldots\right)\right)=s+t n$. Since the values of the other terms of $F(\ldots$, $\left.w_{\imath, i}, \ldots\right)$ are always greater than $s+t n$, we have $v\left(F\left(\ldots, w_{\iota, i}, \ldots\right)\right)$ $=s+t n$, which proves our first assertion. In this argument, we can choose the $u_{\imath, i}$ from $P$. Therefore, if $t=0, w_{\iota, i} \in P$ for $\iota \in \Gamma$, $i=1,2, \ldots$.

Theorem 5. 1. The following three conditions are equivalent.

(i ) $S_{p}^{n}(\pi) \geq 0$ for all $n \in A-$ o.

(ii) Every higher derivation $\delta=\left\{\delta^{0}=i d_{P}, \delta^{1}, \ldots\right\}$ of $P$ into $P$ with index domain $\Lambda$ is extended to a higher derivation $\partial=\left\{\partial^{0}=i d_{R}, \partial^{1}, \ldots\right\}$ of $R$ into $R$ with index domain $A$.

(iii) The canonical $R$-algebra homomorphism $\hat{\varphi}: \widehat{R \otimes_{P} A^{4}(P)} \longrightarrow$ $\widehat{A^{A}(R)}$ is left inversible.

Proof. We shall prove our theorem in the three steps, (ii) $\Longrightarrow$ (i), (i) $\Longrightarrow$ (iii) and (iii) $\Longrightarrow$ (ii).

(ii) $\Longrightarrow$ (i). If $k$ is perfect, we have $\Delta_{p}^{n}(\pi)=\infty$ for $n=1,2, \ldots$ Hence we may assume that $k$ is an infinite field. Let $n$ be an arbitrary integer in $1-\boldsymbol{o}$. By Lemma 5.1, there exists a set of elements $\left\{w_{\imath, i}\right\}_{\iota \in \Gamma, i=1,2, \ldots}$ in $P$ such that $v\left(F_{n}\left(\ldots, W_{\iota, i}, \ldots\right)\right)=$ min $v$ (coefficient of $F_{n}$ ). Let $\delta=\left\{\delta^{0}, \delta^{1}, \ldots\right\}$ be a higher derivation of $P$ into $P$ with index domain $\Lambda$ such that $\delta^{0}=i d_{P}$ and $\delta^{i} c_{\iota}=w_{\iota, i}$ for $\iota \in \Gamma, i \in \Lambda-o$. Let $\partial=\left\{\partial^{0}=i d_{R}, \partial^{1}, \ldots\right\}$ be an extension of $\delta$ to $R$. Then we have $\left(f^{\prime}(\pi)\right)^{2 n-1} \partial^{n} \pi=F_{n}\left(\ldots, w_{\imath, i}, \ldots\right)$ by Proposition 5. 1. Hence $0 \leq v\left(\partial^{n} \pi\right)=v\left(F_{n}\left(\ldots, w_{\imath, i}, \ldots\right)\right)-(2 n-1) v\left(f^{\prime}(\pi)\right)=$ 
min $v\left(\right.$ coefficient of $\left.F_{n}\right)-(2 n-1) v\left(f^{\prime}(\pi)\right)=\Delta_{P}^{n}(\pi)$, which proves our assertion.

(i) $\Longrightarrow$ (iii). It was proved in $\S 4$ that

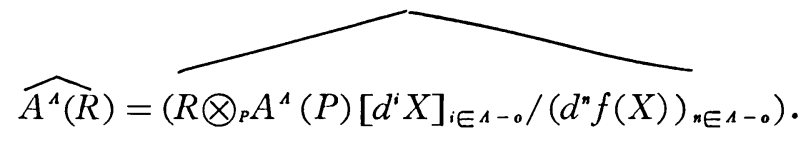

Since $\Delta_{P}^{n}(\pi) \geq 0$ for all $n \in \Lambda$-o, we can define an $R$-algebra homomorphism $\rho: R \otimes_{P} A(P)\left[d^{i} X\right]_{i \in A-0} \longrightarrow \widehat{R \otimes_{P} A^{A}}(P)$ such that $\rho\left(d^{i} c_{\imath}\right)=$ $1 \otimes d_{P}^{i} c_{\imath}$ for $\iota \in \Gamma, i \in \Lambda_{-o}$ and $\rho\left(d^{n} X\right)=\frac{1}{f^{\prime}(\pi)^{2 n-1}} F_{n}\left(\ldots, 1 \otimes d_{P}^{i} c_{\imath}, \ldots\right)$. Then we have $\rho\left(d^{n} f(X)\right)=0$ for every $n \in A-o$. Therefore $\rho$ induces an $R$-algebra homomorphism $\hat{\phi}: \widehat{A^{A}(R)} \longrightarrow \widehat{R \otimes_{P} A^{A}(P)}$ such that $\hat{\phi} \circ \hat{\varphi}=$ the identical homomorphism of $\widehat{R \otimes_{P} A^{A}(P)}$.

(iii) $\Longrightarrow$ (ii). Assume that $\delta$ is given. Since $\delta^{0}=i d_{P_{\text {, }}}$ there exists a $P$-algebra homomorphism $h: A^{A}(P) \longrightarrow P$ such that $\delta^{n}=h \circ d_{P}^{n}$ for $n \in A$. Let $\lambda: R \otimes_{P} A^{\Lambda}(P) \longrightarrow \widehat{R \otimes_{P} A^{4}}(P)$ and $\mu: A^{\Lambda}(R) \longrightarrow \widehat{A^{4}(R)}$ be canonical homorphisms. Since $R$ is complete, $1 \otimes h: R \otimes_{P} A^{A}$ $(P) \longrightarrow R$ induces an $R$-algebra homomorphism $\widehat{\mathbb{\otimes h}:} \widehat{R \otimes_{P} A^{A}(P)}$ $\longrightarrow R$. Let $\hat{\psi}: \widehat{A^{A}(R)} \longrightarrow \widehat{R \otimes_{P} A^{A}(P)}$ be a homomorphism such that $\hat{\phi} \circ \hat{\varphi}=$ identity. We put $g=\widehat{1 \otimes h} \circ \hat{\psi} \circ \mu$. Then we have a commutative diagram:

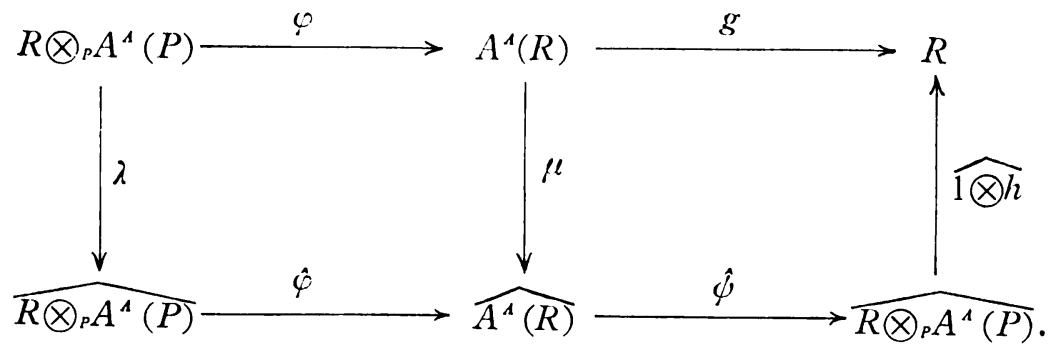

Since $\hat{\phi} \circ \hat{\varphi}=$ identity and $\widehat{1 \otimes h} \circ \lambda=1 \otimes h$, we have $g \circ \varphi=1 \otimes h$. Therefore, the higher derivation $\partial=\left\{\partial^{n}\right\}_{n \in A}$ of $R$ into $R$ with index domain $\Lambda$ such that $\partial^{n}=\operatorname{god}_{R}^{n}(n \in \Lambda)$, is an extension of $\delta$. 
Definition 5. 2 (Neggers [7]). (i) A higher derivation $\partial=$ $\left\{\partial^{0}=i d_{R}, \partial^{1}, \ldots\right\}$ of $R$ into $R$ with index domain $\Lambda$ is called "inducing", if $\partial^{n} m \subset \mathfrak{m}$ for every $n \in \Lambda$, that is, if $\partial$ induces a higher derivation of $k$ into $k$ with index domain $\Lambda$. (ii) $\mathrm{A}$ higher derivation $\bar{\partial}=\left\{\bar{\partial}^{0}=i d_{k}, \bar{\partial}^{1}, \ldots\right\}$ of $k$ into $k$ is called "induced", if there exists a higher derivation $\partial=\left\{\partial^{0}=i d_{R}, \partial^{1}, \ldots\right\}$ of $R$ into $R$ with index domain $\Lambda$ which induces $\tilde{\partial}$.

Theorem 5. 2. $\Delta_{p}^{n}(\pi) \geq 1$ for all $n \in \Lambda-o$ if and only if every higher derivation $\bar{\partial}=\left\{\widehat{\partial}^{\circ}=i d_{k}, \bar{\partial}^{1}, \ldots\right\}$ of $k$ into $k$ with index domain $\Lambda$ is "induced". In this case, every higher derivation $\partial=\left\{\partial^{0}=i d_{R}, \partial^{1}, \ldots\right\}$ of $R$ into $R$ with index domain $A$ is inducing.

Proof. Assume that $\Delta_{p}^{n}(\pi) \geq 1$ for all $n \in \Lambda-o$. Then the last assertion follows from Proposition 5. 1. Let $\left\{h_{\imath, i}\right\}_{\iota \in \Gamma, i \in A_{-}}$be a set of representatives of $\left\{\hat{\partial}^{i} \bar{c}_{\imath}\right\}_{, \in \Gamma, i \in A-o}$ in $P$. Then, by the corollary to Theorem 3.1, there exists a higher derivation $\delta=\left\{\delta^{0}=i d_{P}, \delta^{1}, \ldots\right\}$ of $P$ into $P$ with index domain $\Lambda$ such that $\delta^{i} c_{\iota}=h_{\iota, i}(\iota \in \Gamma, i \in \Lambda-o)$. By Theorem $5.1 \delta$ is extended to a higher derivation $\partial=\left\{\hat{o}^{0}=i d_{R}\right.$, $\left.\partial^{1}, \ldots\right\}$ of $R$ into $R$ with index domain $A$. Since $\partial$ is inducing and $\partial^{i} c_{\imath}=h_{\iota, i}(\iota \in \Gamma, i \in \Lambda-\boldsymbol{o}), \partial$ induces $\bar{\partial}$. Next, we assume that there exists a positive integer $n \in \Lambda$-o such that $\Delta_{P}^{n}(\pi) \leq 0$. Then $k$ is not perfect, hence it is an infinite field. Hence by Lemma 5. 1, there exists a set of elements $\left\{w_{\iota, i}\right\}_{, \in r, i \in A_{-0}}$ in $R$ such that $v\left(F_{n}\left(\ldots, w_{\imath, i}, \ldots\right)\right)=\min v\left(\right.$ coefficient of $\left.F_{n}\right)$. Let $\bar{w}_{c, i}$ be the class of $w_{\imath,}$ modulo $\mathfrak{m}$ for $\iota \in \Gamma, i \in \Lambda-o$. Let $\bar{\partial}=\left\{\bar{\partial}^{0}=i d_{k}, \bar{\partial}^{1}, \ldots\right\}$ be a higher derivation of $k$ into $k$ with index domain $\Lambda$ such that $\delta^{i} c_{\imath}=\bar{w}_{\imath, i}$ for $\iota \in \Gamma, i \in \Lambda-o$. Assume that there exists a higher derivation $\partial=\left\{\partial^{0}=i d_{R}, \partial^{1}, \ldots\right\}$ of $R$ into $R$ which induces $\bar{\partial}$. Then we have $\partial^{i} c_{\imath} \equiv w_{\imath, i} \bmod$. $n t(\iota \in \Gamma, i \in \Lambda-o)$, hence $v\left(F_{n}\left(\ldots, \partial^{i} c_{\imath}, \ldots\right)\right)=v$ $\left(F_{n}\left(\ldots, w_{c, i}, \ldots\right)\right)=\min v\left(\right.$ coefficient of $\left.F_{n}\right)$. Therefore we have $v\left(\partial^{n} \pi\right)=\Delta_{P}^{n}(\pi) \leq 0$ by Proposition 5. 1, and $\partial$ is not "inducing", which is a contradiction. Hence $\bar{\partial}$ is not "induced". 
As an immediate consequence of Theorem 5.2, we have:

Corollary. The condition that $\Delta_{P}^{n}(\pi) \geq 1$ for all $n \in 1-\boldsymbol{o}$, is independent of the choice of $P$ and $\pi$.

$\S 6$. The case $\Lambda=\mathbf{N}_{0}$.

Lemma 6. 1. The following two conditions are equivalent.

(i ) $\Delta_{P}^{n}(\pi) \geq 0$ for all $n \in \mathbf{N}$.

(ii ) $\Delta_{P}^{n}(\pi) \geq 1$ for all $n \in \mathbf{N}$.

Proof. It is enough to prove that (i) implies (ii). Assume that (i) is true and (ii) is false. Let $n$ be the least positive integer such that $\Delta_{P}^{n}(\pi)=0$. Let $\partial=\left\{\partial^{0}=i d_{R}, \partial^{1}, \ldots\right\}$ be a higher derivation of $R$ into $R$. Then by Proposition 4.1, we have

$$
\begin{aligned}
0= & \partial^{n \bullet} f(\pi)=\sum_{j=1} f_{j !}^{(j)}(\pi) \sum_{\substack{i_{1}+\ldots,+i_{j}=n e \\
1 \leq i_{1}, \ldots, i_{j}}} \partial^{i_{1}} \pi \ldots \partial^{i j} \pi \\
& +p \bar{G}_{n \bullet}\left(\partial^{1} \pi, \partial^{2} \pi, \ldots, \ldots, \partial^{i} c_{\imath}, \ldots\right) .
\end{aligned}
$$

Here, we have $\frac{f^{(j)}(\pi)}{j !} \in \|$ for $j<e$ and

$$
\begin{aligned}
& \frac{f^{(\bullet)}(\pi)}{e !} \sum_{\substack{i_{1}+\ldots+i_{q}=n e \\
i_{1} i_{1}, \ldots, i_{e}}} \partial^{i_{1}} \pi \ldots \partial^{i} \pi=\sum_{\substack{i_{1}+\ldots, i_{e}=n_{e} \\
1 \leq i_{1}, \ldots, i_{1}}} \partial^{i_{1}} \pi \ldots \partial^{i} \pi \\
& =\sum_{\substack{i_{1}+\ldots+i_{i}=n e \\
1 \leq i_{1}, \ldots \ldots i, i \\
\left(i_{1} \ldots \ldots, i_{e}\right) \neq(n, \ldots, n)}} \partial^{i i_{1}} \pi \ldots \partial^{i} \pi+\left(\partial^{n} \pi\right)^{e}
\end{aligned}
$$

In the last expression, all terms except the last term belongs to III, because in each of these terms at least one $i_{h}$ is smaller than $n$ and then $\Delta_{P}^{i h}(\pi) \geq 1$, hence $\partial^{i h} \pi \in \mathfrak{m}$ by Theorem 5.2. Therefore we have $\left(\partial^{n} \pi\right)^{\bullet} \in \mathfrak{m}$, hence $\partial^{n} \pi \in \mathfrak{n}$. On the other hand, since $\Delta_{P}^{i}$ $(\pi) \geq 0$ for all $i \in \mathbf{N}_{0}$, for $w_{\iota,,} \in R \quad\left(\iota \in \Gamma, i \in \mathbf{N}_{0}\right)$ arbitrarily given there exists $\partial$ such that $\partial^{i} c_{\imath}=w_{\imath, i}$ by Proposition 5. 1. Especially, there exists a $\hat{o}$ such that $\partial^{n} \pi \notin m$, because of the assumption that $\Delta_{P}^{n}(\pi)=0$, Lemma 5.1 and Proposition 5.1, which is a contradiction. 
By Theorem 5.1, Theorem 5.2 and the above lemma, we get the following theorem.

Theorem 6. 1. The following five conditions are equivalent.

(i ) $\Delta_{p}^{n}(\pi) \geq 0$ for all $n \in \mathbf{N}$.

(ii) Every higher derivation $\delta=\left\{\delta^{0}=i d_{P}, \delta^{1}, \ldots\right\}$ of $P$ into $P$ is extended to a higher derivation $\partial=\left\{\partial^{0}=i d_{R}, \partial^{1}, \ldots\right\}$ of $R$ into $R$.

(iii) The canonical $R$-algebra homomorphism $\hat{\varphi}: \widehat{R \otimes_{P} A(R)} \longrightarrow \widehat{A(R)}$ is left inversible.

(iv) $\Delta_{p}^{n}(\pi) \geq 1$ for all $n \in \mathbf{N}$.

(v) Every higher derivation $\bar{\partial}=\left\{\bar{\partial}^{0}=i d_{k}, \bar{\partial}^{1}, \ldots\right\}$ of $k$ into $k$ is "induced".

Example 6. 1. If $k$ is perfect, $R$ satisfies conditions in Theorem 6.1 .

Example 6. 2. If $R$ is tamely ramified, that is, if $(e, p)=1, R$ satisfies conditions in Theorem 6.1.

Proof. We show that $\Delta_{P}^{n}(\pi) \geq 1$ by induction on $n$, remembering how we deduced relations (4) from (3) at the begining of $\S 5$. Assume that $\Delta_{P}^{j}(\pi) \geq 1$ for $0<j<n$. Then, $F_{j}\left(\ldots, W_{1, i}, \ldots\right)$ can be written as $\left(f^{\prime}(\pi)\right)^{2 j-1} \pi \bar{F}_{j}\left(\ldots, W_{\imath, i}, \ldots\right)(0<j<n)$. Hence the relation (4) is :

$$
\begin{gathered}
\left(f^{\prime}(\pi)\right)^{2 n-1} d_{R}^{n} \pi=-\left(f^{\prime}(\pi)\right)^{2 n-2} \sum_{j=2} \frac{f^{(j)}(\pi)}{j !} \pi^{j} \sum_{\substack{i_{1}+\ldots,+i j=n \\
1 \leq i_{1}, \ldots, i j}} \bar{F}_{i_{1}} \\
\left(\ldots, d_{R}^{i} c_{\imath}, \ldots\right) \ldots \bar{F}_{i_{j}}\left(\ldots, d_{R}^{i} c_{\imath}, \ldots\right)-p\left(f^{\prime}(\pi)\right)^{2 n-2} \\
G_{n}\left(\pi \bar{F}_{1}\left(\ldots, d_{R}^{i} c_{\imath}, \ldots\right), \ldots ; \ldots, d^{i} c_{\imath}, \ldots\right) .
\end{gathered}
$$

On the other hand, we have $v(p)=e, v\left(\frac{f^{(j)}(\pi)}{j !} \pi^{j}\right) \geq e$ for $2 \leq j \leq e$ and $v\left(f^{\prime}(\pi)\right)=e-1$ because $(e, p)=1$. Hence it is easy to see that 
$\Delta_{p}^{n}(\pi) \geq 1$

\section{$\S 7$. Invariant ideals.}

Lemma 7. 1. Let $T$ be a ring which is complete in the p-adic topology. Let $\lambda$ and $\mu$ be homomorphisms of $P$ into $T$. Assume that both induce the same homomorplism of $P / p P$ into $T / p T$ and $\lambda\left(c_{\text {c }}\right)$ $=\mu\left(c_{1}\right)$ for every $\iota \in \Gamma$. Then it holds that $\lambda=\mu$.

Proof. Since it holds that if $a \equiv b(p), a^{p^{n}} \equiv b^{p^{n}}\left(p^{n+1}\right)$ for every $n>0$ in $T, \lambda$ and $\mu$ induce the same map of $\left(P / p^{n+1} P\right)^{p^{n}}$ into $T / p^{n+1} T$. Let $\bar{c}_{\iota}{ }^{(n)}$ be $c$, modulo $p^{n} P$ for $\iota \in \Gamma$. Since $k=k^{p^{n}}\left[\bar{c}_{\imath}\right]_{\iota \in \Gamma}$, we have $P / p^{n+1} P=\left(P / p^{n+1} P\right)^{p^{n}}\left[\bar{c}_{c}^{(n+1)}\right]_{\in \in \Gamma}$. Hence $\lambda$ and $\mu$ induce the same map of $P / p^{n+1} P$ into $T / p^{n+1} T$ for every $h>0$. Since $T$ is complete, we have $\lambda=\mu$.

Lemma 7.2. Assume that $R$ satisfies conditions in Theorem 6. 1 . Let $\lambda$ be an isomorphism of $P$ onto another coefficient ring $P^{\prime}$ of $R$ such that $\lambda$ induces an identical map on $k$. Then $\lambda$ can be extended to an automorphism of $R$.

Proof. Let $\delta=\left(\delta^{0}, \delta^{1}, \ldots\right)$ be a higher derivation of $P$ into $R$ such that $\delta^{0}=$ the canonical injection of $P$ into $R, \delta^{1} c_{\iota}=\lambda\left(c_{\imath}\right)-c_{\imath} \in p P$ for $\iota \in \Gamma$ and $\delta^{i} c \iota=0$ for $i>1$ and $\iota \in \Gamma$. Then $\mu(b)=\sum_{i=0}^{\infty} \delta^{i} b(b \in P)$ is a convergent sum in the $p$-adic topology in $R$, because the $\delta^{i} b$ are convergent linear combinations of countable monomials of $\delta^{1}$ $c$ 's of order $i$, hence $\delta^{i} b \in p^{i} P$. Hence $\mu$ defines a homomorphism of $P$ into $R$. Since $\mu\left(c_{\imath}\right)=c_{\mathrm{t}}+\left(\lambda\left(c_{\mathrm{r}}\right)-c_{\mathrm{r}}\right)=\lambda\left(c_{\mathrm{t}}\right)$ and $\mu$ induces an identical map on $k$, we have $\lambda=\mu$ by Lemma 7.1. Let $\hat{\phi}$ be a grade preserving $R$-algebra homomorphism of $\widehat{A(R)}$ into $\widehat{R \otimes_{P} A(P)}$ such that $\hat{\phi} \circ \hat{\varphi}=$ identity. Let $g: A(P) \longrightarrow R$ be the $P$-algebra homomorphism such that $\delta^{i}=g \circ d_{p}^{i}$ for $i=0,1,2, \ldots$ Let $\hat{g}$ : $\widehat{R \otimes{ }_{\mathrm{P}} A(P)} \longrightarrow R$ be an $R$-algebra homomorphism induced by $g$. 
Then $\hat{g}\left(\widehat{R \otimes_{P} A^{i}(P)}\right) \subset p^{i} R$ for $i=0,1,2, \ldots$ Let $h: A(R) \longrightarrow \widehat{A(R)}$ be the canonical homomorphism. Then we can define a higher derivation $\partial=\left(\partial^{0}, \partial^{1}, \ldots\right)$ of $R$ into $R$ such that $\partial^{i}=\hat{g} \circ \hat{\psi} \circ h \circ d_{R}^{i}$, $i=0,1,2, \ldots$. Then $\partial$ is an extension of $\delta$ and $\partial^{i} b \in p^{i} R$, for $b \in R$, $i=0,1,2, \ldots$, that is, $\nu(b)=\sum_{i=0}^{\infty} \partial^{i} b$ is a convergent sum. Hence $\nu$ defines a homomorphism of $R$ into $R$. Since $R$ is a complete discrete valuation ring of unequal characteristic, $\nu$ is an isomorphism which is an extension of $\lambda$.

Theorem 7.1. Assume that $R$ satisfies conditions in Theorem 6. 1. Then for each $n \geq 1$, the ideal $\left(f^{\prime}(\pi), \frac{f^{\prime \prime}(\pi)}{2 !}, \ldots, \frac{f^{(n)}(\pi)}{n !}\right)$ of $R$ is indepedent of the choice of $P$ and $\pi$.

Proof. Let $F_{n}$ be a free $R$-submodule of $R \otimes{ }_{s} A_{P}(S)=$ $R\left[d_{s / \mathrm{P}}^{1} X, d_{s / \mathrm{P}}^{2} X, \ldots\right]$ generated by monomials of $d_{s / \mathrm{P}}^{i} X$ of weight $n$. Let $H_{n}$ be an $R$-submodule of $F_{n}$ generated by elements of the form:

$$
\left(d_{s / P}^{m} f\right)(\pi) M \ldots \ldots \ldots \ldots \ldots(*)
$$

, where the $M$ are monomials of $d_{s / p}^{i} X$ of weight $n-m \quad(m=1,2$, $\ldots, n)$ and

$$
\left(d^{m} f\right)(\pi)=\sum_{j=1} \frac{f^{(j)}(\pi)}{j !} \sum_{\substack{i_{1}+i_{2}+\cdots+i_{j}=m \\ 1 \leq i_{1}, \ldots, i j}} d_{s / P}^{i_{1}} X \ldots d_{s / P}^{i_{j}} X .
$$

Then we have $A_{P}^{n}(R) \simeq F_{n} / H_{n}$. Let $\kappa_{n}$ be the rank of $F_{n}$, which is independent of the choice of $P$ and $\pi$. Then the $\left(\kappa_{n}-1\right)-t h$ Fittings ideal of $A_{P}^{*}(R)$ is the ideal of $R$ generated by all coefficients of $(*)$. Hence it is $\left(f^{\prime}(\pi), \frac{f^{\prime \prime}(\pi)}{2 !}, \ldots, \frac{f^{(n)}(\pi)}{n !}\right)$. Let $P^{\prime}$ be another coefficient ring of $R$. Then by Lemma 7.2, there exists an automorphism $\nu$ of $R$, inducing the identical map on $k$, such that $\nu$ induces an isomorphism of $P$ onto $P^{\prime}$. Let $\vec{f}(X)$ be an Eisenstein polynomial over $P^{\prime}$ such that $\bar{f}(\nu(\pi))=0$. $\nu$ induces an isomorphism $\rho$ of $A_{P}(R)$ onto $A_{P^{\prime}}(R)$ such that $\rho\left(\mathrm{d}_{R / P}^{i} b\right)=d_{R / P^{\prime}}^{i} \nu(b)$ for $b \in R$. Hence $\rho$ induces an isomorphism between the $(N-1)$-th 
Fittings ideal of $A_{P}(R)$ and that of $A_{P^{\prime}}(R)$, that is, the image of $\left(f^{\prime}(\pi), \ldots, \frac{f^{(n)}(\pi)}{n !}\right)$ by $\nu$ is $\left(\bar{f}^{\prime}(\nu(\pi)), \ldots, \frac{\left.\bar{f}^{(n)}(\nu)(\pi)\right)}{n !}\right)$. Since $R$ is a discrete valuation ring, both ideals are the same one.

The following example shows that even in case $R$ is tamely unramified, the ideal $\left(f^{\prime \prime}(\pi)\right)$ of $R$ is not ncessarily invariant.

Example 7. 1. Assume that $p \neq 2$ and $f(X)=X^{p+1}+p c_{10}$ with $\iota_{0} \in \Gamma$. Then $\left(f^{\prime \prime}(\pi)\right)=\left((p+1) p \pi^{p-1}\right)=\left(\pi^{2 p}\right)$. Let $P^{\prime}$ be another coefficient ring of $R$, containing $c_{\text {c }}$ with $\in \Gamma, \iota \neq \iota_{0}$ and $c_{10}+\pi^{2}$. Then the defining equation of $\pi$ over $P^{\prime}$ is $f_{1}(X)=X^{p+1}-p X^{2}+p$ $\left(c_{10}+\pi^{2}\right)$. Then we have $\left(f^{\prime \prime}(\pi)\right)=\left((p+1) p \pi^{p-1}-p\right)=\left(\pi^{p+1}\right)$. Hence it holds that $\left(f_{1}^{\prime \prime}(\pi)\right) \neq\left(f^{\prime \prime}(\pi)\right)$.

KYOTO UNIVERSITY

\section{References}

[1] R. Berger, Differential höherer Ordnung und Körpererweiterungen bie Primzahlcharakteristik, S.-B. Heidergerger Akad. Wiss. Math. -Natur. Kl. (1966), 143 -202 .

[2] A. Grothendieck, Éléments de Géométrie algébrique IV, Publ. Math. I. H. E. S. (1964).

[3] N. Heerema, Derivations and automorphisms of complete regular local rings, Amer. J. Math. 88 (1966), 33-42.

[4] - Inertial isomorphisms of $v$-rings, Canad. J. Math. 19 (1967), 529539.

[5] _ - Higher derivations and automorphisms of complete local rings, Bull. Amer. Math. Soc. 76 (1970), 1212-1225.

[6] Y. Kawahara and Y. Yokoyama, On higher differentials in commutative rings, Tokyo Sci. Univ. Math. 2 (1966), 12-30.

[7] J. Neggers, Derivations on F-adic fields, Trans. Amer. Math. Soc. 115 (1965), 496-504.

[8] P. Ribenboim, Higher derivations of rings I, II, Rev. Roumaine Math. Pures Appl. 16 (1971), 77-110, 245-272.

[9] S. Suzuki, Some results on Hausdorff m-adic modules and m-adic differentials, J. Math. Kyoto Univ. 2 (1963), 157-182.

[10] Differential modules and derivations of complete discrete valuation rings, J. Math. Kyoto Univ. 9 (1969), 425-437, its corrections and supplements, ibid. 11 (1971), 377-379. 
[11] On Neggers' numbers of discrete valuation rings, J. Math. Kyoto Univ. 11 (1971), 373-375. 